Babin E, Heutte N, Grandazzi G, Prévost V, Robard L. Qualité de vie des patients et soins de support en cancérologie des VADS. Bull Cancer 2014 ; 101 : 505-10. Doi : 10.1684/bdc.2014.1960.

\title{
Qualité de vie des patients et soins de support en cancérologie des VADS
}

\author{
Emmanuel Babin ${ }^{1,2,3}$, Natacha Heutte ${ }^{2}$, Guillaume Grandazzi ${ }^{2,3}$, Virginie Prévost ${ }^{2}$, Laetitia Robard ${ }^{1}$ \\ ${ }^{1} \mathrm{CHU}$ de Caen, Service ORL et chirurgie cervico-faciale, avenue de la Côte-de-Nacre, CS 30001, 14033 Caen \\ cedex 9, France \\ ${ }^{2}$ Inserm U1086, Cancers \& prévention, avenue du Général-Harris, 14076 Caen, France \\ ${ }^{3}$ Université de Caen Basse-Normandie, CERReVEA3918, 14000 Caen, France
}

\section{Résumé}

La qualité de vie des patients pris en charge pour un cancer des voies aéro-digestives supérieures et de leurs accompagnants fait partie des préoccupations actuelles des équipes soignantes. Des outils d'évaluation ont été créés et ont permis de mettre en évidence les répercussions physiques aiguës (douleurs, mucite...) et chroniques (muti-lations, complications post-radiques...) liées à la maladie ou aux différents traitements mais aussi de prendre en considé-ration les répercussions psycho-sociales de cette pathologie. L'amélioration de la qualité de vie passe par une prise en charge réfléchie et globale qui doit associer aux soins soma-tiques, des soins psychiques, une rééducation et une prise en compte des difficultés sociales et de la souffrance des proches. Les soins de support veillent à assurer une bonne qualité de vie aux soignés et leurs proches mais aussi réduire les séquelles physiques liées à la maladie et aux traitements. Ils s'appuient sur une coordination des soins, notamment par les réseaux de cancérologie institués dans le plan cancer, afin d'assurer une prise en charge globale et continue de ces patients.

Mots clés : qualité de vie, cancer de la tête et du cou, soins de support

\begin{abstract}
The quality of life of patients treated for head and neck cancers and their carers is part of the current concerns of health care teams. Assessment tools were created and helped to highlight the severe physical effects (pain, mucositis...) and chronic (mutilation, post-radiation complications...) related to the disease or to different treatments but also to consider the psy-chosocial impact of this disease. Improving the quality of life through a thoughtful and comprehensive support that must be associated with somatic care, mental health care, rehabilitation and inclusion of social difficulties and suffering relatives. Supportive care shall ensure a good quality of life for patients treated and their families but also reduce the physical effects associated with the disease and treatment. They rely on coordination of care including the cancer networks established in the cancer plan to ensure comprehensive and continuous care for these patients.
\end{abstract}

Key words: quality of life, head and neck cancer, supportive care 


\section{Introduction}

Les cancers des voies aéro-digestives et leurs traitements sont à l'origine de complications et de séquelles multiples sur le quotidien de vie des patients. Le retentissement fonctionnel physique est connu par des praticiens. II se mesure sur la douleur, la déglutition, la phonation et la respiration. Les impacts psychologiques et sociaux étaient jusqu'à maintenant plus souvent méconnus.

La quantité de vie gagnée ne constitue plus la seule préoccupation principale des médecins. L'espérance de vie limitée, avec des issues thérapeutiques entraînant rarement la guérison ou a contrario un allongement de la durée de la vie liée à amélioration des traitements et la mobilisation des proches sont à l'origine de l'émergence et de l'évolution de la thématique qualité de vie (QdV).

\section{Définition de la qualité de vie}

L'Organisation mondiale de la santé (OMS) a défini la QdV comme " la perception qu'a un individu de sa place dans la vie, dans le contexte de la culture et du système de valeur dans lequel il vit, en relation avec ses objectifs, ses attentes, ses normes et ses inquiétudes. C'est un concept très large qui peut être influencé de manière complexe par la santé physique du sujet, son état psychologique et son niveau d'indépendance, ses relations sociales et sa relation aux éléments essentiels de son environnement » [1]. En résumé, la QdV est une mesure subjective, qui répond à la perception de soi dans le monde (soi social).

\section{Outils d'évaluationde la qualité de vie [2]}

L'évaluation de la QdV en cancérologie ORL est essentiellement quantitative. L'approche qualitative reste peu développée.

De multiples échelles quantitatives existent. Elles doivent répondre à trois critères : validité, fiabilité et sensibilité [3]. Les différentes échelles de QdV se décomposent en deux groupes : les échelles génériques et les échelles spécifiques. Les échelles génériques (EuroQol, SF-36, PGI) s'intéressent à la QdV et à l'état de santé en dehors d'une pathologie donnée. Ces échelles permettent de comparer la QdV de groupes de patients souffrant de pathologies différentes ou de comparer la QdV de patients souffrant d'une pathologie avec des populations de référence non malades.

Les échelles spécifiques s'intéressent à une maladie (l'échelle EORTC QLQ-C30 pour le cancer, Fact-H\&N pour le cancer ORL...), à un domaine et/ou symptôme (fatigue, dépression, xérostomie, dysphagie...) ou à un traitement (chirurgie ou radiothérapie). Ces échelles sont plus sensibles aux variations cliniques et aux effets des traitements que les échelles globales.

Des échelles peuvent cibler des organes ou des symptômes : la voix avec Vocal Handicap Index (VHI/VHI10), les mucites, la xérostomie et la déglutition avec le Swallowing Quality-of-Life Questionnaire (SWALQOL), l'alimentation avec le Head and Neck Performance Status Scale (PSS-HN), les pathologies dentaires avec le Geriatric Oral Health Assessment Index (GOHAI), la sexualité avec le Modified Sexual Adjustment Questionnaire for Head and Neck Cancer (SAQ), les addictions avec Alcohol Use Disorder Identification Test (AUDIT). Quelques échelles spécifiques à l'anxiété, à la dépression et au retentissement psychique sont validées en français (Hospital Anxiety and Depression Scale [HADS], Beck Depression Inventory [BDI]).

Des échelles destinées à l'évaluation des aidants spécifiques sur l'impact des soins existent : une seule est validée en français (Caregiver Quality of Life Index-Cancer [CQOLC]). L'approche nutritionnelle en cancérologie s'est développée et affinée. En effet, la dénutrition est associée à une augmentation de la 
morbi-mortalité ; elle conduit à une moindre efficacité des thérapeutiques, à une majoration de leur toxicité et impacte sur la QdV des patients [4]. À côté des index anthropométriques classiques (poids, taille, indice de masse corporelle, pli tricipital) et des paramètres biologiques usuels (albuminémie, transthyrétine, protéine C réactive, le Prognostic Inflammatory and Nutritional Index [PINI]), d'autres évaluations ont été développées comme celles de la prise alimentaire avec calcul des ingesta (risque de dénutrition si ingesta $<25 \mathrm{kcal} / \mathrm{kg} / \mathrm{j}$ en cancérologie des VADS, l'apport calorique quotidien souhaitable est de 1600 à $2400 \mathrm{kcal}$, l'apport azoté de l'ordre de 1,25 à 1,85 g de protides/kg/j), l'estimation de la prise alimentaire à partir de la description, par le sujet, de sa prise alimentaire des $24 \mathrm{~h}$ précédentes, l'agenda alimentaire (recueil prospectif, par écrit, sur plusieurs jours, des données des prises alimentaires avec estimation des portions ou du poids des aliments). L'article de V. Prevost et M.C. Grach propose des scores nutritionnels avec notamment Mini Nutritional Assessment (MNA) et Subjective Global Assessment (SGA) [5].

Cette échelle subjective validée en français apprécie le degré de dénutrition sur l'intensité de la perte de poids, la gravité des signes digestifs et cliniques de dénutrition, l'altération fonctionnelle et associe une notion d'intensité du stress métabolique éventuel.

L'auteur répertorie des scores clinicobiologiques et des indices de risque qui aident dans le diagnostic et le suivi nutritionnel.

\section{Quotidien de vie des patients atteints d'un cancer des VADS}

\section{Généralités}

Les cancers des VADS et leurs traitements altèrent la QdV des patients. De multiples facteurs sont cités mais leur interprétation doit rester prudente car les designs des études et les outils utilisés ne sont pas toujours comparables. Néanmoins, quelques généralités peuvent être données. La QdV des patients atteints d'un cancer des VADS reste altérée les premiers mois après l'institution des traitements. Elle s'améliore à distance pour se stabiliser à un an. Le sexe masculin, les patients jeunes, la vie en couple et la poursuite d'une activité professionnelle seraient des facteurs protecteurs de la QdV [6].

Le statut socioéconomique, le niveau éducatif et culturel apparaissent protecteurs de la QdV : les classes défavorisées les plus fréquemment touchées par ce type de cancer consultent souvent tardivement par méconnaissance ou par crainte du diagnostic. La prise en charge implique des traitements "lourds", source de complications et séquelles [7]. La poursuite des addictions (alcool et tabac), connues pour favoriser la genèse de ces cancers, concourt à l'aggravation de la QdV même si elle peut être vécue comme une échappatoire. Les douleurs somatiques et neuropathiques constituent des facteurs majeurs d'altération de la QdV. Elles sont retrouvées chez $50 \%$ des patients avant le traitement, $80 \%$ pendant le traitement et $70 \%$ après le traitement [8]. Un tiers des patients décrivent des douleurs myofaciale, tendineuse et articulaire avec au premier rang les scapulalgies par atteinte du nerf accessoire secondairement à un évidement ganglionnaire cervical. Les douleurs nerveuses sur le trajet des nerfs crâniens mais aussi à type de décharge électrique lors de la rotation cervicale ou de brûlures résultent le plus souvent de la chirurgie et d'une radiothérapie supérieure à 60 Gy.

La moitié des patients traités évalue une Eva supérieure à sept [8]. L'asthénie est un symptôme retrouvé durant tout le temps de la prise en charge des patients. Elle culmine pendant la phase aiguë et s'estompe à distance des traitements. À l'instar de la QdV, ce marqueur est à rapprocher de l'état d'esprit de l'individu et de multiples facteurs socioculturels interviennent. 


\section{Répercussions physiques}

Les cancers des VADS altèrent la respiration, la phonation et la déglutition. Ces trois fonctions sont touchées de manière variable selon le stade évolutif de la maladie et selon les traitements institués avec souvent une combinaison de chirurgie, radiothérapie et/ou chimiothérapie.

Les altérations sont aiguës, chroniques, précoces et tardives. Leurs répercussions varient selon l'état clinique et nutritionnel du patient mais aussi des ressources familiales mises à disposition des patients.

Symptômes liés à la maladie

La localisation des lésions sur les VADS est à l'origine de symptômes variables. La dysphagie est associée à des lésions de l'oropharynx et de l'hypopharynx. La dyspnée et la dysphonie relèvent davantage de lésions laryngées. L'otalgie réflexe peut révéler un cancer du sinus piriforme ou de l'amygdale. Les autres symptômes sont divers et variables avec des saignements buccaux, des mobilités dentaires. L'impossibilité de positionner une prothèse dentaire doit faire rechercher une lésion du palais.

\section{Complications liées aux traitements}

\section{Complications aiguës}

La chirurgie et ses suites constituent dans le domaine de la cancérologie des VADS un facteur important pouvant grever la QdV des patients. En phase aiguë, les infections du site opératoire peuvent atteindre des taux de 10 à $45 \%$ [9]. Les infections respiratoires atteindraient un peu plus de3\%des patients [10]. Les hémorragies par rupture carotidienne surviennent davantage après chirurgie post-radique. Leur taux a diminué avec l'adjonction de lambeaux pédiculés ou libres sur le site opéré. Les communications entre la cavité buccale (orostomes) ou le pharynx (pharyngostomes) et la peau sont des entraves à la reprise de I'alimentation. Elles sont estimées respectivement à $12 \%$ et entre 9 et $25 \%$ [11]. Elles s'accompagnent d'un prolongement de l'hospitalisation et de soins locaux itératifs. L'attente (pour la sortie et l'alimentation per os) est plus ou moins bien vécue et grève la QdV immédiate des patients et des familles.

La radiothérapie est source de mucite aiguë dont le début apparaît en moyenne un mois après le début du traitement, s'estompe un mois après la fin de celui-ci pour disparaître après cinq mois. Si la mucite est retrouvée dans plus de la moitié des cas après radiothérapie standard, elle est quasi constante après les associations radio-chimiothérapie. Un grade élevé (II-IV) de mucite est retrouvé dans un tiers des cas après radiothérapie standard et chez plus de la moitié des patients après modification ou association de radiothérapie [12].

La radiodermite est proportionnelle au risque d'exposition cutanée à l'irradiation. Plusieurs phases peuvent être individualisées avec un érythème, une dépilation, une ulcération voire une nécrose. La douleur est souvent importante et exacerbée au toucher et au moment des soins.

Des rash cutanés existent surtout avec l'association des rayons à une thérapie ciblée (cétuximab). Le traitement chimiothérapique est un facteur d'altération de la QdV. Les principaux effets connus sont les nausées, vomissements (mieux contrôlés avec la prescription d'antagonistes aux récepteurs de la 5hydroxytriptamine et d'antagonistes de récepteurs à la neurokinine), les troubles rénaux et hématotoxiques. L'alopécie liée au docétaxel reste mal vécue. 


\section{Complications chroniques}

La trachéotomie et la trachéostomie post-chirurgie laryngée constituent des éléments majeurs d'altération de la QdV. L'orifice cervical ouvert vers l'extérieur est le siège potentiel de bronchorrhée, d'infections respiratoires et de saignements mais avant tout de troubles vocaux. La privation de la voix naturelle nécessite de recourir à une réhabilitation vocale (voix trachéo-œsophagienne, voix oroœsophagienne, laryngophone) dont le temps d'acquisition est variable. Ce symptôme nuit à la communication avec autrui et est plus ou moins bien vécu.

L'atteinte corporelle liée à cette ouverture cervicale génère des troubles psychiques et sociaux importants [13]. Les séquelles cutanées et esthétiques sont aussi des stigmates qui exposent les patients à la marginalisation.

Les troubles de la déglutition sont liés à différents facteurs : la malocclusion, les difficultés de mastication et surtout l'altération des nerfs crâniens impliqués dans la motricité (XII, X, IX, VII) et la sensibilité (X, IX) du carrefour aéro-digestif. Les chirurgies partielles ou totales du pharyngolarynx exposent à des altérations variables et retentissent sur la QdV des individus d'autant plus si la chirurgie est complétée d'une radiothérapie. Les patients sont souvent contraints de conserver des sondes naso-gastriques ou des gastrostomies d'alimentation plus ou moins longtemps.

La xérostomie constitue une doléance fréquente. Elle apparaît dès lors que la dose d'irradiation dépasse 35-40 Gy ce qui est une constante en cancérologie des VADS [14]. Cette sécheresse buccale modifie le comportement alimentaire des patients. Elle nuit à la phonation et peut gêner la respiration avec la présence persistante de sécrétions collantes pharyngo-laryngées. Elle est source de dysgueusie, favorise les infections locales (candidoses, sous-maxillites, parotidites) et les caries dentaires. L'ostéo-radionécrose constitue une complication majeure de l'irradiation à l'origine de douleurs et troubles de l'alimentation.

L'atteinte du derme à distance de la radiothérapie peut générer une atrophie cutanée, une fibrose, des changements de pigmentation avec l'apparition de télangiectasies. Le syndrome de fibrose radio-induite atteint la peau, les muscles et les nerfs, et constitue l'évolution tardive du traitement radiothérapique. L'expression clinique est visuelle avec les rétractions cutanéo-tendineuses (trismus), fonctionnelles mais aussi douloureuses (neuropathies). La chirurgie des évidements ganglionnaires cervicaux et notamment la section du nerf accessoire est source d'altération fonctionnelle et douloureuse. Le " shoulder syndrome " peut associer : douleur (imputable à la pression passive exercée sur les autres muscles par la chute de l'épaule), déformation (décollement de la scapula, chute du moignon de l'épaule) ou encore limitation de l'amplitude articulaire en abduction [15].

L'œdème post-radique se manifeste par l'apparition d'un lymphœdème (jabot) sous-mentonnier et d'un œdème laryngé gênant la respiration. L’hypoacousie post-radique est un phénomène présent dans $40 \%$ des cas et se manifeste par une atteinte transmissionnelle (otite séreuse ou dysfonctionnement tubaire) ou neurosensorielle par lésions de l'oreille interne [16]. Les atteintes endocriniennes sont avant tout représentées par l'hypothyroïdie qui varie de 50 à $89 \%$ à cinq ans (après une combinaison chirurgie et radiothérapie)[17].

La chimiothérapie génère des complications diverses au long cours : neurologiques (neuropathies périphériques), néphrologiques (insuffisance rénale), otologiques (surdité de perception) et hématologiques avec atteinte des lignées cellulaires. Des troubles sexuels peuvent être rapportés [18]. 


\section{Répercussions psychiques [19]}

Les diverses atteintes corporelles, physiques, esthétiques et fonctionnelles générées par les cancers des VADS et les traitements sont source d'impact émotionnel majeur [20].

Les mutilations, défigurations et modifications du fonctionnement corporel et de l'expression verbale entraînent des changements dans les perceptions et l'image que le patient a de son corps, affectant ainsi ses interactions sociales, sa sexualité et son bien-être émotionnel [21]. La convivialité des repas est souvent perturbée et source d'isolement. En effet, la dysphagie et les douleurs nécessitent souvent une alimentation artificielle par gastrostomie ou nutrition parentérale qui rompt avec les convenances traditionnelles des repas familiaux. Les complications générées par la chirurgie et la radiothérapie sont multiples : fistules oropharyngées, pharyngostomes, nécroses, douleurs, et sont d'autant plus mal tolérées qu'elles sont souvent révélatrices d'un mauvais état nutritionnel ou d'une intoxication alcoolique et tabagique. Les troubles phonatoires et respiratoires potentialisent ces atteintes psychologiques car ils contraignent et stigmatisent : soins de canule, réapprentissage de la respiration, la déglutition, la mastication, la parole. Tout cela survient généralement au sein d'un réseau social fragile et d'un statut économique précaire.

La majorité des patients est associée à un habitus alcolo-tabagique et à des conditions socioéconomiques défavorables (emploi peu qualifié, chômage, instabilité professionnelle) qui génèrent une détresse émotionnelle et une altération de la QdV [22].

Toutefois, la détresse psychologique rencontrée chez les patients atteints de cancer de la sphère ORL reste difficile à évaluer $[23,24]$ : les premiers indicateurs de cette détresse sont la présentation d'un visage triste, un repli sur soi, une colère, une insatisfaction. La prévalence de difficultés psychiatriques dans cette population est décrite chez jusqu'à $66 \%$ des patients [25]. La prévalence des syndromes dépressifs est estimée entre 6 et $15 \%$ [26]. Le risque suicidaire existe [27]. L'humeur dépressive est souvent présente en phase pré-thérapeutique [25]. Les symptômes dépressifs augmentent tout au long du traitement par radiothérapie [28]. Dans l'étude de Humphris, les taux de dépression culminent trois mois après le début du traitement et restent stables jusqu'à sept mois [29]. Les symptômes peuvent persister après la fin des traitements ( $8 \%$ des cas), 18 mois après la fin de la radiothérapie dans une étude récente de Neilson et al.[30].

Les scores de dépression apparaissent fortement liés à la QdV des patients mais ne sont prédits de façon indépendante ni par l'âge ou le sexe, ni par la localisation, le stade ou le type de traitement de la tumeur $[28,31]$. En revanche, la détresse émotionnelle élevée en début de traitement est apparue fortement prédictive de la survenue d'une symptomatologie dépressive après le traitement [32]. Après la fin des traitements, près d'un quart des patients continuerait à fumer, près de la moitié consommerait encore de l'alcool au moins deux fois par semaine sans qu'il soit possible de déterminer si ce comportement additif freinerait le risque dépressif [33]. Au total, le retentissement psychique lié aux cancers des VADS menace la QdV des patients.

La prise en charge doit être réfléchie et globale. Elle doit associer aux soins somatiques, des soins psychiques, une rééducation et une prise en compte des difficultés sociales et de la souffrance des proches. 


\section{Répercussions sociales}

Les cancers de la tête et du cou sont responsables de handicaps fonctionnels transitoires ou permanents (altération ou suppression de la voix physiologique, troubles de la fonction respiratoire, de la déglutition, perte du goût et/ou de l'odorat). Ils créent une rupture biographique dans la vie quotidienne des malades, une métamorphose corporelle et identitaire avec des retentissements psychologiques et sociaux importants [7]. Les taux d'incapacité de travail, chez les patients atteints d'un cancer de la tête et du cou, varient de 34 à $52 \%$ [34]. La perte d'activité professionnelle découle de cette situation. La maladie et les traitements impactent la sexualité des couples et engendrent des modifications de vie (vie commune mais chambres à part, divorces). Les mutilations physiques comme la trachéotomie et la gastrostomie accentuent ces transformations de vie.

Le vécu des patients atteints d'un cancer des VADS est unique et mérite d'être approfondi.

Avec la connaissance du cancer, le patient prend pleinement conscience de son statut de mortel. La maladie et les traitements dans les stades avancés sont à l'origine de la bascule du patient dans le monde de l'invalidité. L'individu malade devient un handicapé. Il est stigmatisé. Le " stigmate » est défini par E. Goffman comme " un attribut que l'on a et que 'on aimerait bien ne pas posséder » [35]. Dans l'exemple du laryngectomisé total, l'individu est porteur d'une double " tare » : il a perdu sa voix originelle et affiche un trou au milieu du cou (trachéostome).

Le stigmate génère une transformation de son identité personnelle (le patient ne se reconnaît plus) et de son identité sociale (les autres le considèrent comme un être différent de celui qu'il était). Cette métamorphose, illustrée dans le texte de F. Kafka, suscite une perte des repères habituels lors des rencontres entre valides et invalides. Le patient est marginalisé et souvent infériorisé. II est victime d'un handicap d'apparence : il n'a nullement perdu ses fonctions intellectuelles, mais tout concourt à le considérer comme un être inférieur. La résultante de ces situations est de favoriser un isolement voire un auto-enfermement du malade.

Longtemps ignorés, les proches des patients peuvent également subir indirectement les modifications familiales, sociales et professionnelles engendrées par le cancer. Les conjoints sont les personnes qui accompagnent le plus souvent ces malades au quotidien et leur QdV apparaît altérée [36].

\section{Soins de support}

Les soins de support sont définis comme " l'ensemble des soins et soutiens nécessaires aux personnes malades tout au long de la maladie conjointement aux traitements spécifiques » [37].

Cette organisation centrée autour du patient et des proches implique une connaissance de la cancérologie par tous les acteurs de soins et la mise en place d'une permanence des soins. Idéalement, la coordination de cette entité s'appuie sur les réseaux de cancérologie institués avec le plan cancer. L'objectif affiché est d'assurer une bonne $\mathrm{QdV}$ aux soignés et ses proches. Les experts veillent à réduire les séquelles physiques liées à la maladie et aux traitements (chirurgie, radiothérapie, chimiothérapie). La douleur, l'asthénie, les troubles nutritionnels sont au premier plan. De même, les experts veillent aussi à lutter contre les troubles digestifs, respiratoires et génito-urinaires, les troubles moteurs et les handicaps, les problèmes odontologiques. 
Une aide psychologique est essentielle et doit être accessible à tous. Enfin, il convient de développer le lien social en gommant les stigmates et la marginalisation engendrés en incitant notamment au retour à une activité professionnelle. L'accompagnement de fin de vie reste le stade ultime de cette prise en charge. In fine, les maîtres mots de cette organisation reposent au sein d'un même territoire de santé, sur l'anticipation et le repérage des situations à risque ainsi que la coordination entre la ville et l'hôpital dans un esprit de prise en charge globale $[38,39]$.

\section{Perspectives, conclusion}

L'amélioration de la QdV des patients s'est développée avec la mise en place, via les plans cancers, de réunions de concertation disciplinaires, de consultations d'annonce médicales et infirmières, de l'amélioration de l'information avec la remise des programmes personnalisés des soins. Les réseaux oncologiques régionaux favorisent la transmission et préviennent l'isolement des patients et des familles.

La multiplication des programmes d'éducation thérapeutiques constitue une étape complémentaire dans l'optique de l'amélioration de la QdV des patients et des proches. De même, le vécu après un cancer constitue une autre étape. Dans cette perspective l'Inserm UMR 912 élabore une enquête nationale sur les survivants du cancer à deux ans du diagnostic.

L'INCa pilote l'étude en partenariat avec les trois principaux régimes d'Assurance maladie (CNAMTS, MSA et RSI). Cette observation descriptive permettra de définir des axes d'amélioration de vie des patients.

\section{Références}

1. OMS Working Group. WHOQOL, measuring quality of life, 1997.

2. Heutte N, Plisson L, Lange M, Prevost V, Babin E. Quality of life tools in head and neck oncology. Eur Ann Otorhinolaryngol Head Neck Dis 2014 ; 131 : 33-47.

3. Sayed SI, Elmiyeh B, Rhys-Evans P, et al. Quality of life and out-comes research in head and neck cancer: a review of the state of the discipline and likely future directions. Cancer Treat Rev 2009 ; 35 : $397-$ 402.

4. Heutte N, Plisson L, Prévost V, Babin E. Qualité de vie en cancérologie ORL [Internet]. Rapport de la Société franc, aise d'ORL et chirurgie cervico-faciale 2013. Paris : Elsevier Masson, 2013 (p. 1-11).

5. Prevost V, Grach MC. Nutritional support and quality of life in cancer patients undergoing palliative care. Eur J Cancer Care (Engl) 2012 ; 21 : 581-90.

6. Babin E, Joly F, Vadillo M, Dehesdin D. [Oncology and quality of life. Study in head and neck cancer]. Ann Otolaryngol Chir Cervicofac $2005 ; 122$ : 134-41.

7. Babin E, Sigston E, Hitier M, Dehesdin D, Marie JP, Choussy O. Quality of life in head and neck cancers patients: predictive factors, functional and psychosocial outcome. Eur Arch Otorhinolaryngol $2008 ; 265$ : 265-70.

8. Navez M, David A, Timochenko A, Prades JM. Les douleurs à point de départ ORL : quelles douleurs, quelle prise en charge ? Douleur Analg $2012 ; 25$ : 144-50. 
9. Lee DH, Kim SY, Nam SY, Choi SH, Choi JW, Roh JL. Risk factors of surgical site infection in patients undergoing major oncological surgery for head and neck cancer. Oral Oncol $2011 ; 47$ : 528-31.

10. Lin HW, Bhattacharyya N. Contemporary assessment of medical morbidity and mortality in head and neck surgery. Otolaryngol Head Neck Surg 2012 ; 146 : 385-9.

11. Righini C, Atallah I, Reyt E. Orostomes et pahryngostomes. Rapport de la Société française d'ORL et chirurgie cervico-faciale. Paris, 2013 (p. 79).

12. Trotti A, Bellm LA, Epstein JB, et al. Mucositis incidence, severity and associated outcomes in patients with head and neck cancer receiving radiotherapy with or without chemotherapy: a systematic literature review. Radiother Oncol $2003 ; 66: 253-62$.

13. Babin E. Le cancer de la gorge et la laryngectomie : la découration. Paris : L'Harmattan, 2011.

14. Jellema AP, Doornaert P, Slotman BJ, Rene Leemans C, Langen-dijk JA. Does radiation dose to the salivary glands and oral cavity predict patient-rated xerostomia and sticky saliva in head and neck cancer patients treated with curative radiotherapy? Radiother Oncol 2005 ; 77 : 164-71.

15. Nahum AM, Mullally W, Marmor L. A syndrome resulting from radical neck dissection. Arch Otolaryngol $1961 ; 74: 424-8$.

16. Jereczek-Fossa BA, Zarowski A, Milani F, Orecchia R. Radiotherapy-induced ear toxicity. Cancer Treat Rev 2003;29: 417-30.

17. Thorp MA, Levitt NS, Mortimore S, Isaacs S. Parathyroid and thyroid function five years after treatment of laryngeal and hypopharyngeal carcinoma. Clin Otolaryngol $1999 ; 24$ : 104-8.

18. Albert S, Guigay J. Séquelles de la chimiothérapie. Rapport de la Société française d'ORL et chirurgie cervico-faciale. Paris, 2013 (p. 137-144).

19. Dauchy S, Reich M. Prise en charge des séquelles psychologiques. Rapport de la Société française d'ORL et chirurgie cervico-faciale. Paris, 2013.

20. Singer S, Krauss 0 , Keszte J, et al. Predictors of emotional distress in patients with head and neck cancer. Head Neck 2012 ; 34 : 180-7.

21. Katz MR, Irish JC, Devins GM, Rodin GM, Gullane PJ. Psychosocial adjustment in head and neck cancer: the impact of disfigurement, gender and social support. Head Neck $2003 ; 25$ : 103-12.

22. Chaukar DA, Walvekar RR, Das AK, et al. Quality of life in head and neck cancer survivors: a crosssectional survey. Am J Otolaryngol $2009 ; 30: 176-80$.

23. Bertrand Deligne J, Chene J. [Psychological management of patients with head and neck cancer]. Ann Otolaryngol Chir Cervi-cofac 1996 ; 113 : 294-8.

24. Breitbart W, Holland J. Psychosocial aspects of head and neck cancer. Semin Oncol $1988 ; 15$ : 619.

25. Kugaya A, Akechi T, Okuyama T, et al. Prevalence, predictive factors, and screening for psychologic distress in patients with newly diagnosed head and neck cancer. Cancer $2000 ; 88: 2817-23$. 
26. Archer J, Hutchison I, Korszun A. Mood and malignancy: head and neck cancer and depression. J Oral Pathol Med 2008;37: 255-70.

27. Hem E, Loge JH, Haldorsen T, Ekeberg $\varnothing$. Suicide risk in cancer patients from 1960 to 1999. J Clin Oncol $2004 ; 22$ : 4209-16.

28. Sehlen $\mathrm{S}$, Lenk $\mathrm{M}$, Herschbach $\mathrm{P}$, et al. Depressive symptoms during and after radiotherapy for head and neck cancer. Head Neck 2003 ; 25 : 1004-18.

29. Humphris GM, Rogers S, McNally D, Lee-Jones C, Brown J, Vaughan D. Fear of recurrence and possible cases of anxiety and depression in orofacial cancer patients. Int J Oral Maxillofac Surg $2003 ; 32$ : 486-91.

30. Neilson K, Pollard A, Boonzaier A, et al. A longitudinal study of distress (depression and anxiety) up to 18 months after radiotherapy for head and neck cancer. Psychooncology $2013 ; 22: 1843-8$.

31. Hassanein KAAM, Musgrove BT, Bradbury E. Psychological out-come of patients following treatment of oral cancer and its relation with functional status and coping mechanisms. J Craniomaxillofac Surg $2005 ; 33: 404-9$.

32. Karnell LH, Funk GF, Christensen AJ, Rosenthal EL, Magnuson JS. Persistent post-treatment depressive symptoms in patients with head and neck cancer. Head Neck 2006 ; 28 : 453-61.

33. Duffy SA, Terrell JE, Valenstein M, Ronis DL, Copeland LA, Connors M. Effect of smoking, alcohol, and depression on the qua-lity of life of head and neck cancer patients. Gen Hosp Psychiatry $2002 ; 24$ : 140-7.

34. Roger $\mathrm{V}$, De Raucourt $\mathrm{D}$, Babin $\mathrm{E}$. Réinsertion et surveillance médicale du laryngectomisé. $E M-$ Consulte [20-710-A-30]. Doi : 10.1016/S0246-0351(13)47001-0.

35. Goffman E. Stigmates. Les usages sociaux des handicaps. Paris : Ed de Minuit, 1977.

36. Cuny F, Babin E. Impact des cancers de la tête et du cou sur la sociabilité des couples. Rapport de la Société française d'ORL et chirurgie cervico-faciale. Paris, 2013.

37. Krakowski I, Boureau F, Bugat R, et al. For a coordination of the supportive care for people affected by severe illnesses: proposition of organization in the public and private health care centres. Bull Cancer $2004 ; 91: 449-56$.

38. DHOS. Les soins de support dans le cadre du plan cancer, 2004.

39. Ministère des Solidarités, de la Santé et de la Famille. Circulaire no DHOS/SDO.2005/101 du 22 février 2005 relative à l'organisation des soins en cancérologie, 2005. 